\title{
Vaccination strategy to target lysyl oxidase-like 4 in dendritic cell based immunotherapy for head and neck cancer
}

\author{
JAN BERND WEISE ${ }^{1}$, KATALIN CSISZAR $^{4}$, STEFAN GOTTSCHLICH $^{1}$, MARKUS HOFFMANN $^{1}$, \\ ANDRÉ SCHMIDT $^{1}$, UTE WEINGARTZ ${ }^{2}$, ILSE ADAMZIK ${ }^{3}$, AXEL HEISER ${ }^{5}$, \\ DIETER KABELITZ ${ }^{2}$, PETRA AMBROSCH ${ }^{1}$ and TIBOR GÖRÖGH ${ }^{1}$ \\ ${ }^{1}$ Department of Otorhinolaryngology, Head and Neck Surgery, ${ }^{2}$ Institute of Immunology, and \\ ${ }^{3}$ Institute of Transfusion Medicine, University of Kiel, Germany; ${ }^{4}$ John A. Burns School of Medicine, \\ University of Hawaii, Honolulu, HI 96822; ${ }^{5}$ Department of Urology, College of Medicine, Gainesville, FL 32608, USA
}

Received August 3, 2007; Accepted October 1, 2007

\begin{abstract}
Overexpression of lysyl oxidase (LOX) is associated with the invasive potential of metastatic breast and head and neck cancer (HNC) cells and reduced metastasis-free and overall survival. Recently, we have demonstrated up-regulation of a new member of the LOX family, lysyl oxidase-like 4 (LOXL4), in invasive HNC revealed a significant correlation between LOXL4 expression and local lymph node metastases and higher tumour stages. The objective of this study was to examine whether cellular LOXL4 may provide an effective target for cell-meditated immunotherapy in invasive tumours associated with LOXL4 overexpression. As a feasibility study we expressed LOXL4 mRNA in immature dendritic cells derived from human peripheral blood mononuclear cells (PBMC). LOXL4 protein expression was ascertained using Western blotting and immunocytochemistry with polyclonal rabbit anti-LOXL4 antibody. The successfully transfected immature dendritic cells (DCs) were induced to mature with GM-CSF, IL-4, IL-1ß, TNF- $\alpha$, IL-6, and PGE2, and then used to stimulate $\mathrm{T}$ cell enriched non-adherent fraction of PBMC. LOXL4 specific T cell stimulation induced cytotoxic $\mathrm{T}$ lymphocyte (CTL) response was monitored using IFN- $\gamma$ secretion from the non-adherent PBMC fraction exposed to mature, LOXL4 transfected DCs acting as the antigen presenting target cells. LOXL4-DC stimulated T cells produced higher IFN- $\gamma$ secretion compared to unstimulated $\mathrm{T}$ cells and $\mathrm{T}$ cells stimulated with untransfected DCs, in the presence of the pan-DR-epitope (PADRE). These initial results demon-
\end{abstract}

Correspondence to: Dr Jan B. Weise, Department of Otorhinolaryngology, Head and Neck Surgery, University of Kiel, ArnoldHeller-Str. 14, D-24105 Kiel, Germany

E-mail: jweise@hno.uni-kiel.de

Key words: dendritic cells, lysyl oxidase-like 4, head and neck cancer, vaccine, immunotherapy strated the potential for LOXL4-transfected DCs to serve as efficient tumour vaccine and support their suitability as a vaccination strategy applicable to cancer patients with tumour specific up-regulation of LOXL4.

\section{Introduction}

A number of cancer-related antigens have been identified to contain epitopes that can be recognized by specialized immune cells, predominantly by cytotoxic $\mathrm{T}$ lymphocytes (CTL). The possibility of tumour-specific therapy has led to continued efforts to develop antigen specific immunotherapeutic strategies for different malignancies.

The essential role of the extracellular matrix (ECM) amine oxidase, lysyl oxidase (LOX), in breast tumour cell invasion was reported from our and other laboratories $(1,2)$. We have also demonstrated that inhibition of LOX expression and catalytic activity reduced the migratory and invasive potential of these cells. Additional evidence indicates that this active LOX induced FAK/Scr/paxillin mediated mechanism was active in other invasive tumour cell types including uveal melanoma cell lines (FAK/Scr) (1) and astrocytes (FAK/ paxillin) (3). Critical in vivo role for LOX in invasive breast tumours was further supported by data in a mouse model for breast cancer (4) and our studies of breast cancer patients (5).

LOX is a copper dependent amine oxidase that catalyzes the oxidation of lysine to semialdehyde, the precursor to the covalent cross linkages that stabilizes elastin and collagen fibers in the ECM $(6,7)$. Micro- and tissue array and a prospective head and neck cancer studies have shown the expression of LOX to be elevated in hypoxic HNC (8). Additionally, LOX expression was found associated with lower distant metastasis-free survival and reduced overall survival in HNC patients (4). Furthermore, LOX regulation by hypoxiainducible factor and association with hypoxia was reported in human breast and HNC $(4,9)$. Inhibition of LOX activity eliminated metastasis in mice with breast tumours indicating that LOX is a candidate therapeutic target for preventing and treating metastases.

Multiple studies reported tumour-associated expression for other members of the LOX family, including LOXL (10), 
LOXL2, LOXL3 and LOXL4 (2). We have found elevated LOXL4 in head and neck squamous cell carcinoma (HNSCC) compared to normal squamous epithelium (11). Results of our recent study demonstrated an overexpression of the LOXL4 transcript in $74 \%$ of invasive HNSCC and $90 \%$ of both primary and metastatic HNSCC cell lines tested, and a significant correlation between LOXL4 expression and local lymph node metastases versus primary tumour types and higher tumour stages (12). Immunocytochemistry demonstrated cellular overexpression of the LOXL4 protein that correlated with the increased mRNA transcription in HNSCC cells. These results strongly support an important role for LOXL4 in the molecular pathogenesis of invasive head and neck carcinomas and demonstrate that both LOX and LOXL4 are potential immunotherapeutic targets .

Bone marrow-derived plasmacytoid and myeloid dendritic cells (DCs) are the most potent antigen-presenting cells capable of activating native $\mathrm{T}$ cells (13-15). Loading $\mathrm{DCs}$ ex vivo with tumour antigens in the form of mRNA can stimulate potent anti-tumour immunity in tumour-bearing mice $(16,17)$. Suggestive evidence of clinically related responses was first seen in phase I clinical trials in patients with prostate cancer immunized with prostate-specific antigen mRNA transfected DCs and in patients with renal cancer immunized with autologous tumour RNA transfected DCs (18-20). Since then, many patients have been treated with antigen-loaded DCs in different tumour types with varying protocols for the generation of DCs and application of the vaccine. While these therapies did not yet fully materialize their potential, all DC vaccines proved to be safe. In this feasibility study, we transfected cloned LOXL4 mRNA into immature dendritic cells and examined whether cellular expression of LOXL4 may provide an effective target for cell-mediated immunotherapy.

\section{Materials and methods}

Human subjects. Peripheral blood mononuclear cells (PBMC) were harvested from healthy volunteers using peripheral blood leukapheresis. All cells used in these experiments were obtained from human subjects, following informed consent through protocols approved by the ethics committee at our institution (AZ: A128/01; Kiel, Germany).

DC generation from peripheral blood. For DC culture, we adopted techniques described by Romani et al (21) and Morse et al (22) and implemented some modifications, particularly in the use of serum-free media and supplements. Briefly, a concentrated leukocyte fraction was generated through a 2-h restricted peripheral blood leukapheresis processing 6-8 1 of blood with each collection. The leukapheresis product was further separated by density-gradient centrifugation over polysucrose/sodium diatrizoate (Histopaque; Sigma Diagnostics, St. Louis, MO, USA), and cells were resuspended in serum-free AIM-V medium (Life Technologies, Grand Island, NY, USA). PBMC were incubated at $2 \times 10^{8}$ cells $/ 30 \mathrm{ml}$ in $\mathrm{T}-150$ culture flasks in a humidified atmosphere for $2 \mathrm{~h}$ at $37^{\circ} \mathrm{C}$ to allow plastic adherence. The adherent cells were used for further incubation in serum-free AIM-V medium supplemented with recombinant human IL-4 (500 U/ml) and recombinant human GM-CSF (800 U/ml; both R\&D Systems, Minneapolis, MN, USA) for 7 days.

Flow cytometry. Immature and mature DCs were characterized by flow cytometry. Briefly, $3 \times 10^{5}$ cells in $100 \mu 1$ PBS were incubated with $5 \mu \mathrm{l}$ each of monoclonal antibodies or isotype controls (Caltag, Hamburg, Germany) for $20 \mathrm{~min}$ in the dark at room temperature. Cells were washed three times in PBS and analysed by flow cytometry (FACSCalibur, Becton Dickinson, Heidelberg, Germany) and with the CellQuest/WinMDI software. DC preparations fulfilling certain phenotypic criteria as outlined in the results section were used for subsequent experiments.

Cloning strategy and sequence analysis. A purified doublestranded full length LOXL4 cDNA fragment was ligated into the pCR2.1 Topo cloning vector and amplified in E. coli competent cells during overnight incubation. Plasmid DNA was extracted and prepared for cycle-sequencing reaction by PCR using Dye Terminator (Applied Biosystems, Weiterstadt, Germany). Sequencing was performed using the M13 primer binding sites of the pCR2.1 Topo vector. The reaction mixture contained $1 \mu \mathrm{g}$ plasmid-DNA, 10 pmol M13 upstream and downstream-primer mix, 5\% (v/v) dimethylsulfoxide, and $4 \mu 1$ premix (Applied Biosystems) in a total volume of $20 \mu 1$. PCR was performed for 25 cycles at $55^{\circ} \mathrm{C}$ for $15 \mathrm{sec}$, and $60^{\circ} \mathrm{C}$ for $4 \mathrm{~min}$ (two-step-annealing). After amplification the PCR product was extracted using absolute ethanol, redissolved in TSR-buffer (Applied Biosystems) and subjected to sequencing (ABI PRISM-310, Applied Biosystems). The sequence obtained was compared with sequences deposited in the Human Gene Bank Database, NCIB (accession no. AY036093).

Generation of LOXL4/pSp73/A64/NotI and in vitro transcription of LOXL4 $m R N A$. To generate in vitro transcribed LOXL4 mRNA, the LOXL4 cDNA was subcloned into the vector $\mathrm{pSP73/A64/NotI,} \mathrm{kindly} \mathrm{provided} \mathrm{by} \mathrm{David} \mathrm{Boczkowski}$ (23). A 2277-bp fragment containing the entire coding sequence for LOXL4 was isolated from pCR2.1 Topo vector. LOXL4 cDNA was excised and inserted into pSp73/A64/ NotI using HindIII (A'AGCT_T) and XhoI (C'TCGA_G). Subcloning generated the plasmid LOXL4/pSp73/A64/NotI, which contains the Amp ${ }^{\circledR}$, a T7 promoter and the PolyAsequence. After linearization with SpeI, mRNA was generated by in vitro transcription with T7 RNA polymerase (Ambion mMessage mMachine kit, Austin, TX, USA). As a control RNA encoding jelly fish green fluorescent protein (eGFP) was used in some experiments.

RNA transfection and maturation of human DC. Transfection of immature DCs with RNA was performed by electroporation (Gene Pulser II, Bio-Rad Laboratories, Munich, Germany). In brief, DCs were washed twice in PBS, counted, and centrifuged at $300 \mathrm{x} \mathrm{g}$ for $10 \mathrm{~min}$. Subsequently, DCs were resuspended at a concentration of $1 \times 10^{7}$ cells $/ \mathrm{ml}$ in Opti-MEM medium (Invitrogen, Life Technologies, Karlsruhe, Germany). mRNA $(10 \mu \mathrm{g})$ (eGFP mRNA or LOXL4 mRNA) and $2 \times 10^{6}$ immature DCs were mixed in Opti-MEM for a $0.4 \mathrm{~cm}$ fusion chamber (Bio-Rad Lab.). Electroporation was performed 
under various conditions. The best electroporation results (high transfection rate, viability of cells $\geq 80 \%$ within the first $2 \mathrm{~h}$ ) were obtained using the following settings: $0.6 \mathrm{kV} / \mathrm{cm}$, $260 \mathrm{mV}, 0 \%$ modulation, $40 \mathrm{~Hz}$, one square-wave pulse of $16 \mathrm{~ms}$. Subsequently, cells were allowed to rest for $5 \mathrm{~min}$ before transfer into fresh culture medium for a 2 -h reconstitution phase in a humidified incubator at $37^{\circ} \mathrm{C} / 5 \% \quad \mathrm{CO}_{2}$. Viability of cells was assessed by trypan blue dye exclusion in a hematocytometer before and 2, 24 and $48 \mathrm{~h}$ after electroporation.

Maturation of the transfected immature DCs was performed by culturing for 1 day in serum-free medium supplemented with GM-CSF, IL-4, IL-1ß, TNF- $\alpha$ (10 ng/ml; CellConcepts), IL-6 (1000 U/ml; CellConcepts), and PGE2 (1 $\mu \mathrm{g} / \mathrm{ml}$; SigmaAldrich, Deisenhofen, Germany).

Western blot analysis and immunocytochemistry. For Western blot analysis, DCs were homogenised and cell lysates were subjected to SDS-PAGE. After electrophoresis the proteins were transferred to positively charged nitrocellulose membranes (Millipore, Eschborn, Germany).

For immunocytochemistry, DCs were fixed with ice-cold methanol for 10 min and blocked with PBS (pH 7.3) containing $1 \%(\mathrm{w} / \mathrm{v}) \mathrm{BSA}$ (Sigma) for $1 \mathrm{~h}$ at room temperature. A primary affinity purified polyclonal rabbit anti-LOXL4 antibody from our own production (12) was added to the cell preparations for $1 \mathrm{~h}$ at room temperature, followed by incubation with a biotin-conjugated swine anti-rabbit IgG secondary antibody (Dako, Hamburg, Germany) at room temperature for $1 \mathrm{~h}$. The optimum concentration of the primary antibody (1:400) was determined using serial dilutions of highly purified synthetic peptide (Eurogentec, Seraing, Belgium). When diluted 1:400, the antibody detected LOXL4 at a minimal concentration of $0.1 \mu \mathrm{g} / \mathrm{ml}$ in Western blotting, and stained a single band in the Western blot analysis of total protein extracts from the carcinoma cells. Negative controls were obtained by omission of the primary antibody and by incubating the primary antibody with specific blocking peptide in 5-10-fold molar excess before staining.

Generation of human LOXL4 antigen specific T cells and $I N F-\gamma$ secretion assay. LOXL4 specific T cells were generated using a protocol adopted from Heiser et al (18); in brief, the $\mathrm{T}$ cell-enriched non-adherent fraction of PBMC obtained after the DC plastic adherence step was used. Non-adherent PBMC were cultured in serum-free medium supplemented with recombinant human IL-2 (20 U/ml; CellConcepts) and recombinant human IL-7 (10 ng/ml; CellConcepts). Cells were stimulated weekly at least four times with autologous LOXL4-mRNA-transfected DCs in absence or presence of the pan-DR-epitope (PADRE, Pharmexa, Denmark) in two separate experiments.

Target cells (LOXL4-mRNA-transfected DC, unstimulated $\mathrm{T}$ cells or untransfected DC in absence or presence of PADRE) were incubated with CTL at E:T ratio of 100:1, 30:1 and 10:1 in 96-well U-bottom plates for $12 \mathrm{~h}$ at $37^{\circ} \mathrm{C}$ in $5 \% \mathrm{CO}_{2}$. Subsequently, $150 \mu 1$ of supernatant was harvested and used in cytokine secretion assay and analysed by FACS array according to manufacturer's protocol (CBA FLEX system, BD Biosciences, San Jose, USA).
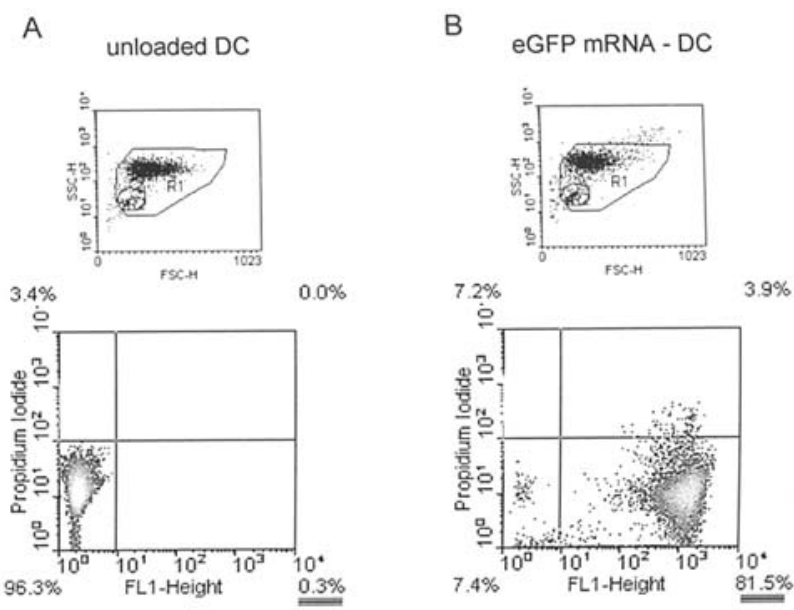

Figure 1. Transfection of immature DCs with RNA using electroporation technique. High transfection rates of viable DCs, electroporation protocol was established with eGFP mRNA, and eGFP protein expression was analyzed by flow cytometry. (A) Control DCs (unloaded DCs, left) compared with (B) transfected DCs (right) reveal transfection rate of $>80 \%$. Result for a representative experiment is shown.

\section{Results}

In vitro transcription of the LOXL4 $m R N A$. To clarify that the LOXL4 sequence derived from the LOXL4/pCR2.1 clone was accurate, plasmid DNA was sequenced. The sequencing data revealed the full coding and additional 440 bp noncoding sequences at both ends of the LOXL4 mRNA. Both end sequences exhibited identity to human LOXL4 cDNA (Accession no. AY036093). In order to produce LOXL4 mRNA, the cloning vector LOXL4/pSP73-Sph/A64/NotI was linearized with SpeI and in vitro transcription was performed using T7 RNA polymerase. The resulting material was subjected to gel electrophoresis that revealed two bands of expected size for the plasmid and the LOXL4 mRNA.

RNA transfection of DCs. Transfection of mRNA into DCs was performed using electroporation. In order to establish the efficiency of electroporation, mRNA encoding eGFP was used. Successful transfection resulted in translation of the mRNA by the DCs resulting in fluorescent eGFP expression, that was detected using flow cytometry (Fig. 1). Optimized electroporation using reproducible settings resulted in a transfection rate of $>80 \%$.

Using the optimized settings, LOXL4 mRNA was successfully transfected into immature DCs. LOXL4 transfected DCs showed the same viability as GFP transfected DCs. Expression of the LOXL4 protein in DCs cells was analyzed by two different methods. Western blot analysis of cell extracts revealed expression of the correct size LOXL4 in DCs (Fig. 2A, lane 2). LOXL4 expression and cellular localization was further confirmed by immunocytochemical (ICC) staining using polyclonal rabbit anti-LOXL4 in transfected DCs (Fig. 1B). The majority of cells displayed a positive cytoplasmatic ICC staining, and no staining of nontransfected DCs. Non-transfected DCs on Western blotting and ICC (Fig. 2A) using an unrelated control antibody and secondary antibody alone were negative for LOXL4 staining (data not presented). 
A

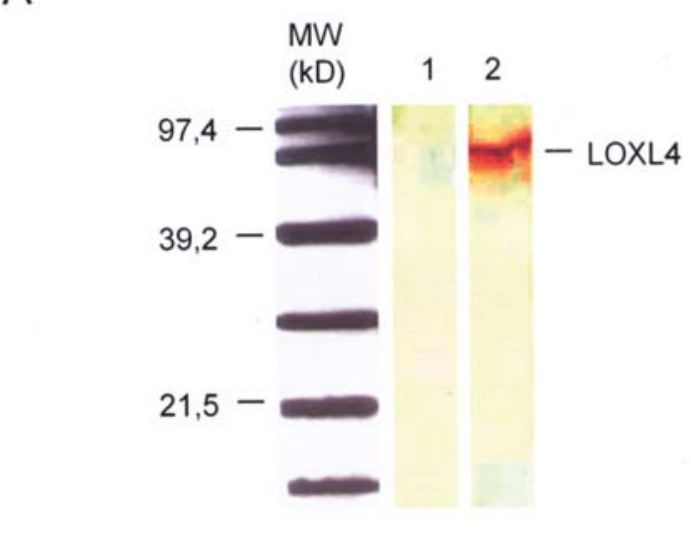

$\mathrm{B} / 1$

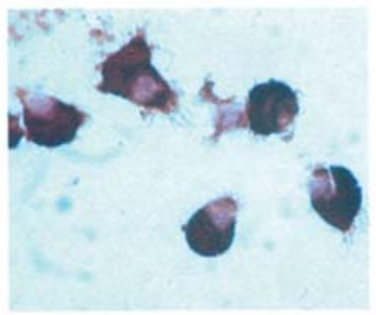

\section{$\mathrm{B} / 2$}

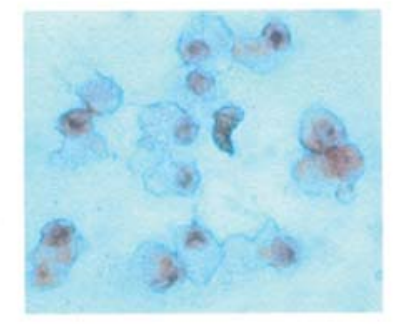

C
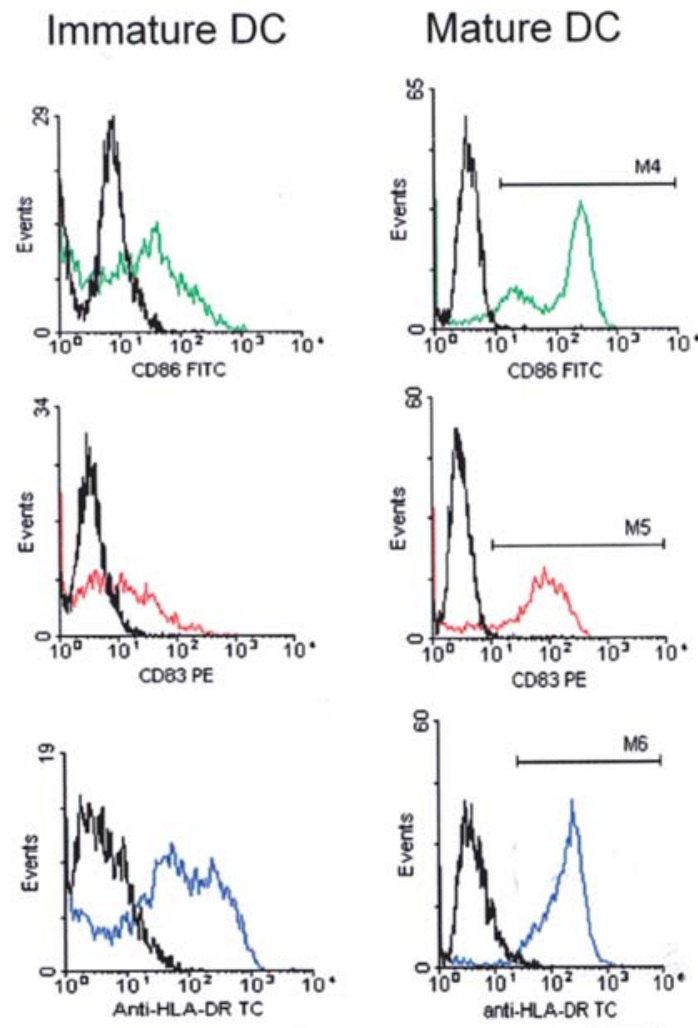

Mature DC
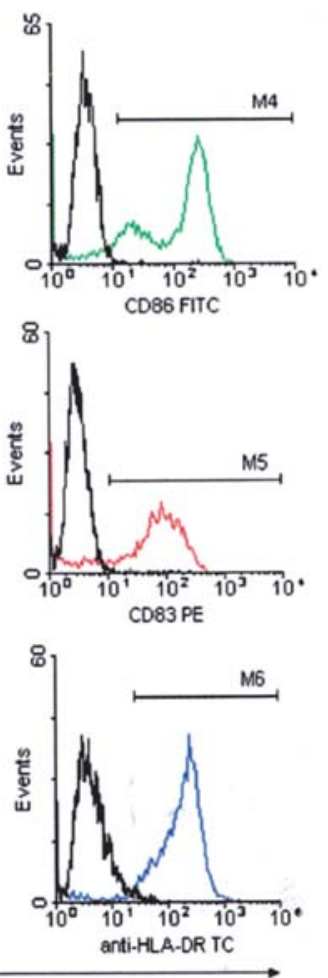

Figure 2. Characterization of LOXL4 mRNA-transfected DCs. (A) Western blotting showing LOXL4 protein ( $~ 80 \mathrm{kDa}) 24 \mathrm{~h}$ after transfection of cells with LOXL4-mRNA (lane 2), and lack of expression in non-transfected DCs (lane 1). (B/1) Immunocytochemical staining with polyclonal rabbit anti-LOXL4 antibody detected cytoplasmatic distribution of LOXL4 in DCs $24 \mathrm{~h}$ after transfection. (B/2) For negative controls, cells were transfected with eGFP-mRNA and nuclei were counterstained with hemalaun (x200). (C) Phenotyping of mature DCs confirmed the up-regulation of HLA-DR and CD86 and expression of CD83 antigens.

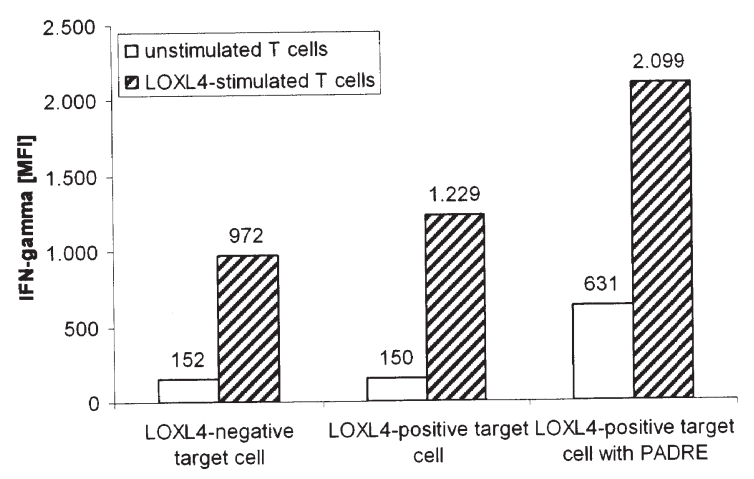

Figure 3. Antigen-presenting capacity of LOXL4-RNA-transfected DCs. INF- $\gamma$ secretion of different effector cells (unstimulated and LOXL4stimulated T cells) are shown. Different targets were compared in the absence or presence of PADRE (pan helper T cell stimulant) with effector to target ratio of 100:1 (MFI, mean fluorescence intensity).

Phenotyping of DCs. Immature DCs were generated from PBMC derived from healthy volunteers. By flow cytometry, DCs were analyzed for the presence of antigens known to be characteristic for monocyte-derived DCs (23). Immature monocyte-derived DCs were positive for HLA-DR, CD1a, CD80, CD86 and negative for CD14 and CD83. After electroporation and maturation using TNF- $\alpha$ and LPS induction, the
DCs displayed enhanced expression of HLA-DR, CD1a, CD80, CD86, and also stained positive for CD40 and CD83 which has been specified to be characteristic profile (14) for mature DCs (Fig. 2C displays data for CD86, CD83 and HLA-DR).

Antigen-presenting capacity of LOXL4-RNA-transfected $D C s$. To evaluate the antigen-presenting capacity of LOXL4RNA-transfected DCs, non-adherent PBMC obtained from healthy volunteers were stimulated with autologous LOXL4RNA-transfected DCs. Subsequently, INF- $\gamma$ secretion, a marker for activated antigen specific $\mathrm{T}$ cells, into the supernatant was determined. Results from these experiments demonstrate that LOXL4-specific T cells have been successfully induced (Fig. 3). Stimulation of non-adherent PBMC with LOXL4RNA-transfected DCs resulted in a $26 \%$ increased INF- $\gamma$ secretion after stimulation with LOXL4-positive target cells compared to unstimulated $\mathrm{T}$ cells and $\mathrm{T}$ cells stimulated with untransfected DCs at E:T ratio of 100:1. The presence of PADRE further enhanced $\mathrm{T}$ helper cell activation increasing INF- $\gamma$ secretion by $116 \%$ compared to LOXL4-negative target cells. Stimulation with LOXL4-RNA-transfected DCs at E:T ratio of 30:1 and 10:1 and stimulation with untransfected DCs failed to stimulate PBMC to secrete INF- $\gamma$. In the presence of PADRE, unspecific increase of INF- $\gamma$ secretion was observed in these controls. 


\section{Discussion}

It is well accepted that DCs are capable of inducing antitumour immunity by priming $\mathrm{T}$ cells to tumour antigens. Many tumour antigens have been identified as potential targets for specific cytotoxic T lymphocytes mediating antitumour immunity. However, most tumour antigens identified in head and neck cancer are self-proteins with expression in the normal mucosa. As the human lysyl oxidaselike protein 4 was not detected in healthy oral epithelia, and up-regulation of LOXL4 expression in invasive HNC was associated with significant correlation to higher tumour stages and metastatases, provide substantial evidence that targeting LOXL4 is a promising approach of DC based vaccination therapy (12).

We successfully transfected LOXL4 mRNA into dendritic cells derived from PBMC, ascertained LOXL4 protein expression in these cells and demonstrated that the LOXL4 expressing DCs stimulate the $\mathrm{T}$ cell enriched non-adherent fraction of PBMC. Using INF- $\gamma$ secretion assay for monitoring LOXL4-specific CTL induction our results support the use of LOXL4 mRNA-transfected DCs as a promising vaccination tool for head and neck cancer therapy.

RNA transfection of DCs. Transfer of exogenous genetic material into foreign cells can be accomplished actively by physical or chemical methods or passively by diffusion. The RNA transfection method provides the insertion of genetic information into the cytoplasm for direct translation of genes into proteins without affecting the genome. Therefore this technology, while efficient, does not carry the high risk of gene therapy. The advantages of using mRNA loaded DCs are 3-fold: first, unlike synthetic peptides encoding HLA restricted epitopes, mRNA spanning the entire coding sequence of a protein, LOXL4 in our case, is likely to encode multiple epitopes corresponding to many different HLA-restricted alleles; second, as previously shown, mRNA transfected DCs can function as specific targets in CTL assays and provide a general platform to monitor CTL responses without the need to culture and label tumour cells as targets $(14,17,18,20)$; third, RNA pulsed DC vaccines can be used to stimulate CTL from virtually all cancer patients regardless of their HLA genotype.

LOXL4 as a target tumour antigen and its expression in DCs. We have previously observed significant correlation between LOXL4 expression and regional lymph node metastases versus primary tumour types $(\mathrm{p}<0.01)$ and higher tumour stages $(p<0.01)$. As up-regulated LOXL4 is characteristic for head and neck tumour cells, while its expression is undetected or very low in normal squamous epithelia and similarly low in other normal tissue types $(1,11,12)$, LOXL4 is an excellent candidate tumour antigen for immune therapy for head and neck cancer and potentially for other tumour types over-expressing LOXL4 $(1,2,5)$. The target LOXL4 cDNA, was originally generated and cloned into the pCR2.1 Topo vector (8) that we have modified for our expression needs. We have developed an efficient electroporation protocol using eGFP mRNA monitored by flow cytometry. Our results revealed that the electroporation-based mRNA delivery system was highly efficient and displayed low cytotoxicity against
DCs under optimal electrical settings. These observations were consistent with other reports showing that the electroporationbased mRNA delivery system is superior to the commonly used techniques of lipid based transfection or passive pulsing by providing better mRNA transfection efficiency (24-28). Following the optimized protocol, we have generated LOXL4 electroporated DCs and confirmed the specific expression of the LOXL4 protein in these cells using Western blot analysis and immunocytochemistry. Results from these analyses revealed LOXL4 protein synthesis in DCs $24 \mathrm{~h}$ and $48 \mathrm{~h}$ after cell transfection (Fig. 2).

Maturation of DCs in vivo. The cellular basis of the vaccine, immature DCs were generated and cultured from PBMC. The activation of DCs is a crucial step in the initiation of adaptive immunity because it links peripheral events initiated by the encounter with pathogens to the activation and expansion of antigen-specific $\mathrm{T}$ lymphocytes during their typical migration from the skin to lymphoid tissues. It is well recognized that a wide variety of microbial products and endogenous signals can trigger DC activation $(29,30)$. Most researchers believe that activated DCs applied in vaccines need to be matured in vitro before application to enrich the cell's migration capacity and to avoid the tolerance inducing potential of immature DCs to self-antigens (18,31-33). In our study, maturation was achieved with a cytokine cocktail (GM-CSF, IL-4, IL-1ß, TNF- $\alpha$, IL-6, PGE2) and confirmed by flow cytometry. The resulting mature myeloid DCs expressed higher levels of HLA-DR and CD86 and were CD83 and CD80-positive.

Functional in vitro assessment of the vaccine. RNA-transfected DCs expressing different target antigens have been shown to successfully trigger antigen-specific $\mathrm{T}$ cell responses in vitro. The induction of a strong immune response by antigen specific cytotoxic $\mathrm{T}$ lymphocytes, the effector cells for tumour cell killing, is the primary goal for any vaccination strategy $(29,34)$. In our study, LOXL4-stimulated $\mathrm{T}$ cells showed increased secretion of INF- $\gamma$ when targeting LOXL4-positive T cells. The in vitro stimulation of $\mathrm{T}$ cells with LOXL4-transfected DCs and the resulting induction of LOXL4-specific CTL provided evidence for the potential of these DCs to serve as tumour vaccine. The background signal on LOXL4-negative target cells might be due to the fact that the cells we used have been obtained directly from stimulated cell culture and were still secreting some INF- $\gamma$. As known from preclinical and clinical trials, PADRE induces important co-stimulatory signals that activate helper T cells (35). When combined with LOXL4-stimulation, a PADRE-induced helper T cell response significantly enhanced the LOXL4-specific $\mathrm{T}$ cell response referring to LOXL4 specific CTL induction. This activating effect was not observed at E:T ratio lower than 100:1.

In conclusion, we have demonstrated the successful generation of a DC-based vaccine targeting the novel antigen, LOXL4. Our results provide evidence for the in vitro induction of LOXL4-specific CTL as a functional proof towards the development of LOXL4-based anticancer vaccine. This vaccine may serve as part of therapeutic strategies applicable for cancer patients with tumour cell specific up-regulation of 
the LOXL4 gene, particularly those with advanced stages of HNSCC.

\section{Acknowledgements}

This study was supported in part by grants from IZKF Faculty of Medicine, University of Kiel, Germany.

\section{References}

1. Kirschmann DA, Seftor EA, Fong SF, et al: A molecular role for lysyl oxidase in breast cancer invasion. Cancer Res 62: 4478-4483, 2002.

2. Payne SL, Hendrix MJ and Kirschmann DA: Paradoxical roles for lysyl oxidases in cancer - a prospect. J Cell Biochem 101: 1338-1354, 2007.

3. Laczko R, Szauter K, Jansen MK, et al: Active lysyl oxidase (LOX) correlates with FAK/paxillin activation and migration in invasive astrocytes. Neuropathol Applied Neurol (In press).

4. Erler JT, Bennewith KL, Nicolau M, et al: Lysyl oxidase is essential for hypoxia-induced metastasis. Nature 440: 1222-1226, 2006.

5. Payne SL, Fogelgren B, Hess AR, et al: Lysyl oxidase regulates breast cancer cell migration and adhesion through a hydrogen peroxide-mediated mechanism. Cancer Res 65: 1429-1436, 2005.

6. Kagan M and Li W: Lysyl oxidase - properties, specificity, and biological roles inside and outside of the cell. J Cell Biochem 88: 660-672, 2003.

7. Maki JM, Tikkanen $\mathrm{H}$ and Kivirikko KI: Cloning and characterization of a fifth human lysyl oxidase isoenzyme: the third member of the lysyl oxidase-related subfamily with four scavenger receptor cysteine-rich domains. Matrix Biol 20: 493-496, 2001.

8. Chen Y, Shi G, Xia W, et al: Identification of hypoxia-regulated proteins in head and neck cancer by proteomic and tissue array profiling. Cancer Res 64: 7302-7310, 2004.

9. Erler JT and Giaccia AJ: Lysyl oxidase mediates hypoxic control of metastasis. Cancer Res 66: 10238-10241, 2006.

10. Wu G, Cuo, Z, Chang X, et al: LOXL1 and LOXL4 are epigenetically silenced and can inhibit Ras/extracellular signalregulated kinase signaling pathway in human bladder cancer. Cancer Res 67: 4123-4129, 2007.

11. Holtmeier C, Gorogh T, Beier U, et al: Overexpression of a novel lysyl oxidase-like gene in human head and neck squamous cell carcinomas. Anticancer Res 23: 2585-2591, 2003.

12. Gorogh T, Weise JB, Holtmeier C, et al: Selective upregulation and amplification of the lysyl oxidase-like 4 (LOXL4) gene in head and neck squamous cell carcinoma. J Pathol 212: 74-82, 2007.

13. Steinman RM: Dendritic cells and immune-based therapies. Exp Hematol 24: 859-862, 1996.

14. Nair SK, Boczkowski D, Morse M, Cumming RI, Lyerly HK and Gilboa E: Induction of primary carcinoembryonic antigen (CEA)-specific cytotoxic $\mathrm{T}$ lymphocytes in vitro using human dendritic cells transfected with RNA. Nat Biotechnol 16: 364-369, 1998 .

15. Banchereau J, Briere F, Caux C, et al: Immunobiology of dendritic cells. Annu Rev Immunol 18: 767-811, 2000.

16. Ashley DM, Faiola B, Nair S, Hale LP, Bigner DD and Gilboa E: Bone marrow-generated dendritic cells pulsed with tumour extracts or tumour RNA induce antitumour immunity against central nervous system tumours. J Exp Med 186: 1177-1182, 1997.

17. Zeis M, Siegel S, Wagner A, et al: Generation of cytotoxic responses in mice and human individuals against hematological malignancies using survivin-RNA-transfected dendritic cells. J Immunol 170: 5391-5397, 2003.
18. Heiser A, Maurice MA, Yancey DR, Coleman DM, Dahm P and Vieweg J: Human dendritic cells transfected with renal tumour RNA stimulate polyclonal $\mathrm{T}$-cell responses against antigens expressed by primary and metastatic tumours. Cancer Res 61: 3388-3393, 2001.

19. Heiser A, Coleman D, Dannull J, et al: Autologous dendritic cells transfected with prostate-specific antigen RNA stimulate CTL responses against metastatic prostate tumours J Clin Invest 109: 4009-4017, 2002.

20. Su Z, Dannull J, Heiser A, et al: Immunological and clinical responses in metastatic renal cancer patients vaccinated with tumour RNA-transfected dendritic cells. Cancer Res 63: 2127-2133, 2003.

21. Romani N, Gruner S, Brang D, et al: Proliferating dendritic cell progenitors in human blood. J Exp Med 180: 83-93, 1994.

22. Morse MA, Lyerly HK, Gilboa E, Thomas E and Nair SK: Optimization of the sequence of antigen loading and CD40ligand-induced maturation of dendritic cells. Cancer Res 58: 2965-2968, 1998.

23. Nair SK, Morse M, Boczkowski D, et al: Induction of tumour specific cytotoxic T lymphocytes in cancer patients by autologous tumour RNA-transfected dendritic cells. Ann Surg 235: 540-549, 2002.

24. Kalady MF, Onaitis MW, Padilla KM, Emani S, Tyler DS and Pruitt SK: Enhanced dendritic cell antigen presentation in RNAbased immunotherapy. J Surg Res 105: 17-24, 2002.

25. Minami K, Yamaguchi Y, Ohshita A, et al: Generation of antigen-presenting cells using cultured dendritic cells and amplified autologous tumour mRNA. Oncology 69: 399-407, 2005.

26. Van Tendeloo VF, Ponsaerts P, Lardon F, et al: Highly efficient gene delivery by mRNA electroporation in human hematopoietic cells: superiority to lipofection and passive pulsing of mRNA and to electroporation of plasmid cDNA for tumour antigen loading of dendritic cells. Blood 98: 49-56, 2001.

27. Van Driessche A, Ponsaerts P, van Bockstaele DR, van Tendeloo VF and Berneman ZN: Messenger RNA electroporation: an efficient tool in immunotherapy and stem cell research. Folia Histochem Cytobiol 43: 213-216, 2005.

28. Milano F, van Baal JW, Rygiel AM, et al: An improved protocol for generation of immuno-potent dendritic cells through direct electroporation of $\mathrm{CD} 14^{+}$monocytes. J Immunol Methods 321: 94-106, 2007.

29. Macagno A, Napolitani G, Lanzavecchia A and Sallusto F: Duration, combination and timing: the signal integration model of dendritic cell activation. Trends Immunol 28: 227-233, 2007.

30. Saalbach A, Klein C, Sleeman J, et al: Dermal fibroblasts induce maturation of dendritic cells. J Immunol 178: 4966-4974, 2007.

31. Ponsaerts P, Van Tendeloo VF and Berneman ZN: Cancer immunotherapy using RNA-loaded dendritic cells. Clin Exp Immunol 134: 378-384, 2003.

32. Gilboa E and Vieweg J: Cancer immunotherapy with mRNAtransfected dendritic cells. Immunol Rev 199: 251-263, 2004.

33. Prechtel AT and Steinkasserer A: CD83 - an update on functions and prospects of the maturation marker of dendritic cells. Arch Dermatol Res 299: 59-69, 2007.

34. Alexander J, del Guercio MF, Frame B, et al: Development of experimental carbohydrate-conjugate vaccines composed of Streptococcus pneumoniae capsular polysaccharides and the universal helper T-lymphocyte epitope (PADRE). Vaccine 22: 2362-2367, 2004.

35. Aragoneses-Fenoll L and Corbi AL: Dendritic cells - still a promising tool for cancer immunotherapy. Clin Transl Oncol 9: 77-82, 2007. 